\title{
A review on emerging fungal infections and their significance
}

\begin{abstract}
The incidence of fungal infections is increasing at an alarming rate, presenting an enormous pressure and challenge to healthcare professionals for their diagnosis and treatment. Emerging fungal infections is also cause of significant morbidity and mortality. This emergence is directly related to the growing population of immunecompromised individuals. Patient with condition such as Granulocytopenia, advanced HIV infection, bone marrow and solid organ transplantation, cancer, diabetes mellitus, severe burn and trauma and severe malnutrition are among many others predisposing factor for low immunity. Demographics changes, Microbial adaptation and Technological advances are few factors which promote these emergences. Infection may be caused by various types of fungi such as yeast and mould including hyaline as well as dematiaceous mould. Populations which are not exposed to endemic fungi and are healthy may also are at risk by dimorphic fungi. Increase in surveillance, availability of rapid noninvasive diagnostic test, monitoring of development of resistance to antifungal agents, research on pathogenesis, prevention, and control of fungal infection are few steps we may put together to cope up with these emerging health problems.
\end{abstract}

Keywords: emergence, opportunistic fungi, immunodeficiency, invasive mould, antifungal, public health
Volume I Issue 2 - 2015

\author{
Ravikant, Tanveer Kaur, Satish Gupte, \\ Mandeep Kaur \\ Department of Microbiology, Gian Sagar Medical College \& \\ Hospital, India
}

Correspondence: Dr. Satish Gupte, Department of Microbiology, Gian Sagar Medical College \& Hospital, Rajpura, India, Email drsatishgupte@hotmail.com

Received: July 14, 2015 | Published: September 21, 2015

\section{Introduction}

The emergence of fungi causing human infection is a growing serious public health problem. Fungi of medical importance may be characterized as primary or opportunistic. Primary which causes infection in healthy population who are not exposed to endemic fungi, whereas opportunistic infect immunosuppresed individuals. ${ }^{1}$ State of art medical devices and services increases the depending population on healthcare which leads to increase in number of susceptible hosts. The emergence and re-emergence of fungal infections such as candidiasis, mucormycosis (zygomycosis), aspergillosis, cryptococcosis, and pneumocystosis are quite common. However Candidemia is one of the leading causes of blood-stream infections with mortality rate more than $30 \%^{2}$ whereas Aspergillus can affect more than $45 \%$ of susceptible host. ${ }^{3}$ Zygomycosis is common among diabetics all around the globe including India. ${ }^{4}$ Mortality associated with invasive fungal infections among patients hospitalized in ICUs has reached to $67 \%{ }^{3}$

Addition to the above incidence of invasive fungal infections is rising despite the expanded armamentarium of antifungal agents now available and invasive fungal infections continue to produce significant morbidity and mortality. ${ }^{5}$ Populations are also susceptible to invasive fungal infections because of external pressures from antibiotic usage, which explains why the burden of invasive fungal infections is unfortunately rising commensurately with these medical advances. Emerging of Mycotic Infection in Patients Infected with Mycobacterium tuberculosis also has been reported from India. ${ }^{6}$ This article reviews the epidemiology, factor contributing the emergence of fungal infection.

\section{Factor contributing emergence}

Demographics changes - Increase in number of defenseless hosts and heightened identification of fungi has resulted from both increased awareness and incidences. AIDS epidemic results in increased numbers of a variety of associated fungal disease such as mucocutaneous candidiasis, Pneumocystis carinii pneumonia and cryptococcal meningitis and many others. In highly endemic areas, the prevalence of coccidioidomycosis and histoplasmosis in AIDS patients is as high as $30 \% .{ }^{1}$ Microbial adaptation-The incidence, morbidity and mortality of fungal infections in transplant recipients leads to beginning of clinical trials of various antifungal prophylactic regimens, the development of effective oral antifungal agents has resulted in their widespread use in hospital settings and in the community at large, including over-the-counter use are factor contributing evolution of antifungal resistance and a need for antifungal stewardship. The level of resistance to antifungal agents is relatively low due to the possible absence of drug-resistant plasmid or transposons in fungi. Emergence of intrinsically resistant fungal species as a human pathogen is compounding the challenge of planning treatment strategies. Beyond these confounding factors, the conditions leading to clinical resistance should be kept in mind while managing invasive fungal infections in immune-compromised patients.

Technological advances in the care of critically ill patients including very low birth weight infants are common trend in modern well- equipped clinical facilities which increases patient survival rate, while the use of prophylactic antibiotics, indwelling catheters and prosthetic devices, hyper alimentation, intensive cancer chemotherapeutic regimens, and organ and bone marrow transplants predispose patients to a variety of fungal infections. For example, the incidence of Candida infection in neonates and outbreaks in neonatal intensive care units are increasing. Newer Land use, travel and commerce also promote emergence of fungal infections in immunecompetent persons who are not previously exposed. 


\section{The evolving epidemiology}

Epidemiological trends that have emerged among immune suppressed are an increased incidence of invasive mold infections such as aspergillosis. The emerging invasive fungal infections in the solid-organ transplant recipients are candidiasis $(53 \%$ of all invasive fungal infections found) followed by invasive aspergillosis (19\%), cryptococcosis $(8 \%)$, non-Aspergillus molds $(8 \%)$, endemic fungi $(5 \%)$, and zygomycosis $(2 \%){ }^{8}$ There is increase in incidence of infections caused by non-albicans Candida species such as Candida tropicalis, Candida parapsilosis, and Candida glabrata which are isolated much more frequently as causes of invasive candidiasis worldwide but $C$. albicans still remains the most common isolate. ${ }^{9}$ The emergence of zygomycosis warrants further discussion, however Zygomycetes, Fusarium, Scedosparium, Paceilomyces, Trichoderma, Scopulariopsis, Dematiaceous fungi (exophilia, alternaria, and bipolaris), hromoblastomycosis, Trichosporon, Malassezia, Rhodotorula, penicillum marneffei, Paracoccidioides and Sporothrix are emerging fungal infection among immunosuppresed individuals. ${ }^{10}$

Common fungal pathogens in HIV infection and their most frequent clinical syndromes are

a) Candida albicans: oral Thrush, vaginal candidiasis, esophageal candidiasis.

b) Cryptococcus neoformans: Meningitis and Histoplasma capsulatum -Disseminated infection with fever and weight loss.

c) Coccidioides immitis: Diffuse and focal pulmonary disease.

d) Blastomyces dermatitidis: Localized pulmonary disease and disseminated infection including meningitis.

e) Aspergillus fumigates: Pulmonary disease with fever, cough and hemoptysis, Penicillium marneffei Fever alone or with pulmonary infiltrates, lymphadenopathy, or cutaneous lesions. ${ }^{11}$

Patient with liver or renal insufficiency are prone to $C$. krusei infections with Fluconazole showing high resistance. ${ }^{12}$ There is a vast number of emerging fungal infections which needed to be mentioned but it is not feasible, anyway some them have been mentioned in the following texts. Gastrointestinal basidiobolomycosis is an emerging infection that leads to diagnostic confusion, morbidity and mortality. It can cause intestinal perforation. Diagnosis requires awareness and consideration of its possibility in the differential diagnosis of patients with abdominal masses and eosinophilia mostly among children. ${ }^{13}$

Pseudallescheria boydii/Scedosporium apiospermum and Scedosporium prolificans are emerging opportunistic pathogens among the ever increasing immunocompromised patient population. These organisms are also emerging pathogens among immunocompetent individuals and are the etiologic agents of white-grain mycetoma and keratitis infections, which even when recognized and treated leave individuals with potential severe deficits. These fungal pathogens are distinctively difficult to treat given their inherent resistance to available anti- fungal agents. Isolation, proper identification, and susceptibility testing of the fungal isolates are important steps in the optimal treatment of these infections. While advances in antifungal therapy have been achieved during the past decade, surgical debridement and augmentation of host defenses re- main critical elements in the battle against these organisms. ${ }^{14}$

Paecilomyces lilacinus have a very low biological control potential and pose a medical hazard to researcher, students, and applicators because of the potential mycoses of skin, lungs, heart, and eyes. It also differ widely in virulence and ability to become established in soil. ${ }^{15}$ Trichoderma species are ubiquitous saprophytic fungi commonly found in soil worldwide. Classically, nine species aggregates have been described as members of this genus, with four different species of Trichoderma identified as causes of human disease. Although only rarely pathogenic in humans, Trichoderma spp. have been reported to cause a pulmonary mycetoma, peritonitis, infection of a perihepatic hematoma, a brain abscess, and disseminated disease. These infections occurred in adult immunocompromised patients, some of whom had foreign body implants as significant predisposing factors for opportunistic infections. Treatment required removal of the foreign bodies, surgical intervention when necessary, and antifungal agents. ${ }^{16}$

Rhodotorula are saprophytic yeasts and common isolated in foods and beverages along with peanuts, apple cider, cherries, fresh fruits, fruit juice, cheese, sausages, edible molluscs, and crustaceans. Dermatitis in sea lions, chickens, and cats, and lung infections and otitis in sheep and cattle are few emerging opportunistic infections including bloodstream infections in humans. ${ }^{17}$ The Zygomycete are diverse group of molds causes opportunists cutaneous or wound infections The production of coenocytic hyphae, zygospore production, and often floccose colony morphology help in identification to some extent. Their role in human disease such as subcutaneous mycosis, sinusitis disease, and even disseminated disease are of great concern. ${ }^{18}$ $S$. schenckii is a phylogenic species found in different geographic locations all around the globe are capable of causing cutaneous, subcutaneous and disseminated form of fungal infection among Immunodeficients. It Inhibit melanin formation that protects the fungus from immune system and that can be used as new target for antifungals. ${ }^{19}$

Emerging endemic infections infect all types of people, including those with a normal immune system. Although very much like TB, they cause disease only in specific circumstances. A huge number of individuals are infected, but only a few get diseased. As these infections have a number of properties in common, we should think of them as a group: histoplasmosis, coccioidomycosis, and blastmycosis ${ }^{20}$ such infections are caused by dimorphs. They grow as molds in soil and reproduce there by sporulation. When they enter in a human, which happens exclusively via the respiratory route, they become yeasts. They also have a restricted geographic distribution and finally, and most importantly, they all cause disease with symptoms almost indistinguishable from TB. ${ }^{21}$ Other opportunistic invasive infections are caused by Malassezia spp. Its manifestations are characterized by catheter-associated sepsis related to hyperalimentation with lipid emulsions and pneumonia. Infections due to Fusarium spp. can cause mycetoma, endophthalmitis, facial granuloma, osteomyelitis, and brain abscess. Disseminated disease in neutropenic patients has positive blood cultures and skin lesions. Fungi such as Fusarium species, Trichosporon species, Curvularia species, and Alternaria species previously were thought to represent contamination or harmless colonization when isolated from immunocompromised patients. 22,23

\section{The public health challenge}

With the increase in numbers of individuals with low immunity and people with more susceptibility for fungal infections generate pressure on cost of human life and health care. Surveillance is one of the tools for monitoring emergence of fungal infection and which can also monitor morbidity, mortality, and cost of cases. New disease 
threats should be responded at the earliest irrespective of their origin, wherever they emerge domestically or internationally. People vulnerable to emerging fungal infections may provide the option for the improvement of health care delivery to these populations and may facilitate early substantiation of new fungal disease among population. ${ }^{1}$

True incidences of theses mycoses are not easy to assess as fungal diseases are not nationally notifiable. Even at states level incidence of mycoses are underreported and are not uncommon. There are ecological niches for the different types of endemic mycoses as for example in case of Blastomycosis and Penicilliosis marneffei, one of the leading fungal infections, hospital or region may not be the same. The pathogenic potential of the fungi and the form of disease that results differ according to risk. Drug- resistant strains of the fungi are another threat added to the situation. Understanding exposure and transmission - Fungal diseases are different from many other infectious diseases in that the life-threatening mycoses are generally not communicable from person to person. The endemic mycoses are generally acquired via inhalation of infectious spores from an environmental reservoir, usually soil. Cryptococcosis, Aspergillosis, Coccidioidomycosis, Histoplasmosis, Blastomycosis, Penidilliosis marneffei and PCP are all either known or thought to be transmitted by inhalation of infectious spores, not via person-to-person spread. In contrast, the yeasts Candida, Trichosporon, and Malassezia are normally resident on human skin or in the gastrointestinal tract and pose the potential for person-to-person transmission, particularly in health care settings.

\section{Conclusion}

Fungal diseases were once primarily inconveniences or, rarely, life-threatening illnesses. Because of increasing numbers of immunecompromised hosts and increase in antifungal resistance, the fungi have become important problems in both the hospital and the community, with ever-increasing morbidity, mortality, and economic costs. These emerging pathogens will require greater awareness and a concerted effort by clinicians, researchers, the pharmaceutical industry, and public health officials. A prompt and accurate diagnosis is crucial for a better outcome; therefore a high degree of suspicion is needed, based on the knowledge of risk factors, disease manifestations and local epidemiology.

\section{Acknowledgements}

None.

\section{Conflict of interest}

The author declares no conflict of interest.

\section{References}

1. Dixon DM, McNeil MM, Cohen ML, et al. Fungal infections: a growing threat. Public Health Rep. 1996;111(3):226-235.

2. Wisplinghoff H, Bischoff T, Tallent SM, et al. Nosocomial bloodstream infections in US hospitals: analysis of 24,179 cases from a prospective nationwide surveillance study. Clin Infect Dis. 2004;39(3):309-317.

3. Maschmeyer G, Haas A, Cornely OA. Invasive aspergillosis: epidemiology, diagnosis and management in immunocompromised patients. Drugs. 2007;67(11):1567-1601.

4. Chakrabarti A, Chatterjee SS, Das A, et al. Invasive zygomycosis in India: experience in a tertiary care hospital. Postgrad Med J. 2009;85(1009):573-581.
5. Chian-Yong Low, Coleman Rotstein. Emerging fungal infections in immune-compromised patients. F1000 Med Rep. 2011;3:14.

6. Sunita B, Mahendra R. Emerging of Mycotic Infection in Patients Infected with Mycobacterium tuberculosis. World Journal of Medical Sciences. 2008;3(2):74-80.

7. Chakrabarti A. Drug resistance in fungi - an emerging problem. Regional Health Forum. 2011;15(1):97-103.

8. Pappas PG, Alexander BD, Andes DR, et al. Invasive fungal infections among organ transplant recipients: results of the Transplant-Associated Infection Surveillance Network (TRANSNET). Clin Infect Dis. 2010;50(8):1101-1111.

9. A Mayr, Lass-Flörl C. Epidemiology and antifungal resistance in invasive aspergillosis according to primary disease - review of the literature. Eur J Med Res. 2011;16(4):153-157.

10. Huprikara S, Shohamb S, the AST Infectious Diseases Community of Practice. Emerging Fungal Infections in Solid Organ Transplantation. American Journal of Transplantation. 2013;13:262-271.

11. Ampel NM. Emerging Disease Issues and Fungal Pathogens Associated with HIV Infection. Emerg Infect Dis. 1996;2(2):109-116.

12. Francuzik W, Sklodowska A, Adamska K, et al. Prevalence of Yeast Fungal Infections in Intensive Care Unit in Poland. Peer J PrePrints. 2015;3:e785v1.

13. El-Shabrawi MH, Kamal NM, Jouini R, et al. Gastrointestinal basidiobolomycosis: an emerging fungal infection causing bowel perforation in a child. J Med Microbiol. 2011;60(Pt 9):1395-1402.

14. Cortez KJ, Roilides E, Quiroz-Telles F, et al. Infections Caused by Scedosporium spp. Clin Microbiol Rev. 2008;21(1):157-197.

15. Esser RP, El-Gholl NE. Paecilomyces lilacinus, a fungus that parasitizes nematode eggs. Fla Dept Agric \& Consumer Serv, Division of plant Industry. Nematology circular No. 203. 1993.

16. Munoz FM, Demmler GJ, Travis WR, et al. Trichoderma longibrachiatum Infection in a Pediatric Patient with Aplastic Anemia. J Clin Microbiol. 1997;35(2):499-503.

17. Fernanda W, Luciano ZG. Epidemiology of Rhodotorula: An Emerging Pathogen. Interdiscip Perspect Infect Dis. 2012;2012:456717.

18. Ribes JA, Vanover-Sams CL, Baker DJ. Zygomycetes in Human Disease. Clin Microbiol Rev. 2000;13(2):236-301.

19. Mahajan VK. Sporotrichosis: An Overview and Therapeutic Options. Dermatol Res Pract. 2014;2014:272376.

20. Cohen MS, Isturiz RE, Malech HL, et al. Fungal infection in chronic granulomatous disease. The importance of the phagocyte in defense against fungi. Am J Med. 1981;71(1):59-66.

21. Jensen HE, Salonen J, Ekfors T. The use of immunohistochemistry to improve sensitivity and specificity in the diagnosis of systemic mycoses in patients with haematological malignancies. J Pathol. 1997;181(1):100 105 .

22. Dromer F, Mathoulin S, Dupont B, et al. Epidemiology of cryptococcosis in France: A 9-year survey (1985-1993).French Cryptococcosis Study Group. Clin Infect Dis. 1996;23(1):82-90.

23. Nawange N, Singh SM, Nawange SR, et al. Spectrum of Opportunistic Fungal Infections in Cancer/HIV Patients: Emerging Fungal Pathogens from Jabalpur Madhya Pradesh Central India: Scholars Journal of Applied Medical Sciences (SJAMS). Sch J App Med Sci. 2015;3(3E):1385-1390. 